\title{
Effects of the soft X-ray burst from SN 1987A on its circumstellar medium
}

\author{
Peter Lundqvist ${ }^{1}$ and Claes Fransson ${ }^{2}$ \\ ${ }^{1}$ Lund Observatory, Box 43, S-221 00 Lund, Sweden \\ ${ }^{2}$ Stockholm Observatory, S-133 00 Saltsjöbaden, Sweden
}

\begin{abstract}
The observations of the narrow UV and optical emission lines from SN 1987A are modelled as reprocessed radiation in a shell around the supernova, heated and ionized by the soft $X$-ray and EUV radiation at the shock breakout. Constraints on the early soft $X$-ray burst are discussed, as well as the physical conditions and abundances in the shell.
\end{abstract}

\section{Introduction}

Since May 1987 the spectra of SN 1987A have shown a number of narrow emission lines in the UV (Fransson et al., 1989, hereafter F89). During the first $\sim 400$ days after the explosion, the luminosity of most of the lines (C III] $\lambda 1909, N$ III] $\lambda 1750, O$ III] $\lambda 1664$ and $N$ $V \lambda 1240)$ increased nearly linearly with time, while He II $\lambda 1640$ and $N$ IV] $\lambda 1486$ were nearly constant. After $\sim 400$ days a decline of the line fluxes started (Sonneborn et al., 1988). Narrow optical [O III], He II $\lambda 4686$ and Balmer emission lines have also been observed (Wampler and Richichi 1988). For a more detailed discussion of the observations, we refer to the paper by Panagia in this volume.

The observations provide important constraints on the parameters of the emitting gas. In F89, the electron density of the emitting gas was found to be $\sim 2.6 \times 10^{4} \mathrm{~cm}^{-3}$, and an analysis of the [O III] $\lambda \lambda 4959-5007 / 4363$ ratio gave a gas temperature of $\sim 5 \times 10^{4} \mathrm{~K}, \sim 300$ days after the explosion (Wampler and Richichi 1988). A simple abundance analysis gave $N / C$ and $\mathrm{N} / \mathrm{O}$ ratios corresponding to $\sim 37$ and $\sim 12$ times solar, respectively (F89), indicating that the gas has been CNO-processed. The elapsed time between the explosion and the turnover of the UV line fluxes indicates that the shell radius is $\sim 5 \times 10^{17} \mathrm{~cm}$ (Sonneborn et al. 1988), consistent with the optical determination by Wampler and Richichi.

In this paper we summarize some recent attempts to model this emission. A more detailed discussion of the constraints on the ionizing radiation, may be found in Fransson and Lundqvist (1988), and of the physical conditions of the emitting shell in Lundqvist and Fransson (1989). 


\section{Modelling the narrow emission lines}

Due to the high ionization potential of the UV line emitting ions ( $77 \mathrm{eV}$ for $\mathrm{N} \mathrm{V}$ ), it was in F89 and Lundqvist and Fransson (1987) argued that the most likely source of excitation is the strong burst of EUV radiation expected to occur when the shock wave breaks through the surface of the supernova progenitor. This ionizes and heats the circumstellar gas around the progenitor. The emitting gas is likely to be in the form of a dense, thin shell at a distance, $\sim 5 \times 10^{17} \mathrm{~cm}$ from the supernova. The time evolution of the lines is then determined by a combination of light travel time effects and recombination of the gas. The observed emission at time $t$ is

$$
L_{o b s}=\frac{c}{2 R_{s_{s}}} \int_{t_{\text {min }}}^{t} L_{e}\left(t_{e}\right) d t_{e} .
$$

Here $\mathrm{L}_{\mathrm{e}}\left(\mathrm{t}_{\mathrm{e}}\right)$ is the total emitted luminosity at time $t_{e}$ and $t_{\min }=\max \left(v, t-2 R_{s} / c\right)$. If the emissivity of the gas drops quickly after the initial ionization this becomes $\mathrm{L}_{\mathrm{obs}}=$ constant. If instead the emissivity is constant, $L_{o b s}=c t L_{e} / 2 R_{S}$. Since the emissivity in the different lines is controlled by the recombination and cooling of the gas, a constant flux indicates either that the gas is recombining fast, or that the emissivity drops quickly because of cooling. A linear increase of the flux consequently implies fairly constant conditions of emission.

To test this scenario we have done detailed calculations of the temperature and ionization of the shell. Since the problem is time dependent, we have calculated the evolution of the physical quantities from the initial ionization until the final recombination of the shell. The evolution of the ionizing radiation is from hydrodynamic models of the explosion (Shigeyama et al. 1988; Woosley 1988; Arnett 1988). These show that immediately after the outbreak the effective temperature was $(2-5) \times 10^{5} \mathrm{~K}$, after which the temperature decreased to less than $3 \times 10^{4} \mathrm{~K}$ in $\sim 5$ hours. The time integrated sum of the ionizing radiation from the photosphere has a form quite different from a blackbody, with an excess of soft photons.

As an illustration, we show in Fig. 1 the temperature and ionization in the middle of the shell as a function of time, together with the instantaneous and integrated (i.e. observed) line luminosities from the shell. For this model we have used the spectrum from the Shigeyama et al. 11E1Y 6 model, a density of $2.6 \times 10^{4} \mathrm{~cm}^{-3}$, and a shell distance of $5 \times 10^{17}$ $\mathrm{cm}$. The elements included are $\mathrm{H}, \mathrm{He}, \mathrm{C}, \mathrm{N}, \mathrm{O}$, Ne and $\mathrm{S}$ with abundances $\mathrm{He} / \mathrm{H}=0.1$, $\mathrm{N} / \mathrm{C}=5, \mathrm{~N} / \mathrm{O}=2$ and an overall metallicity of 0.3 times solar. The upper panel of Fig. 1 shows that the temperature immediately after the burst is $1.35 \times 10^{5} \mathrm{~K}$. Hydrogen as well as helium is fully ionized, while most of the metals are in their helium like stages, like $\mathrm{N}$ 


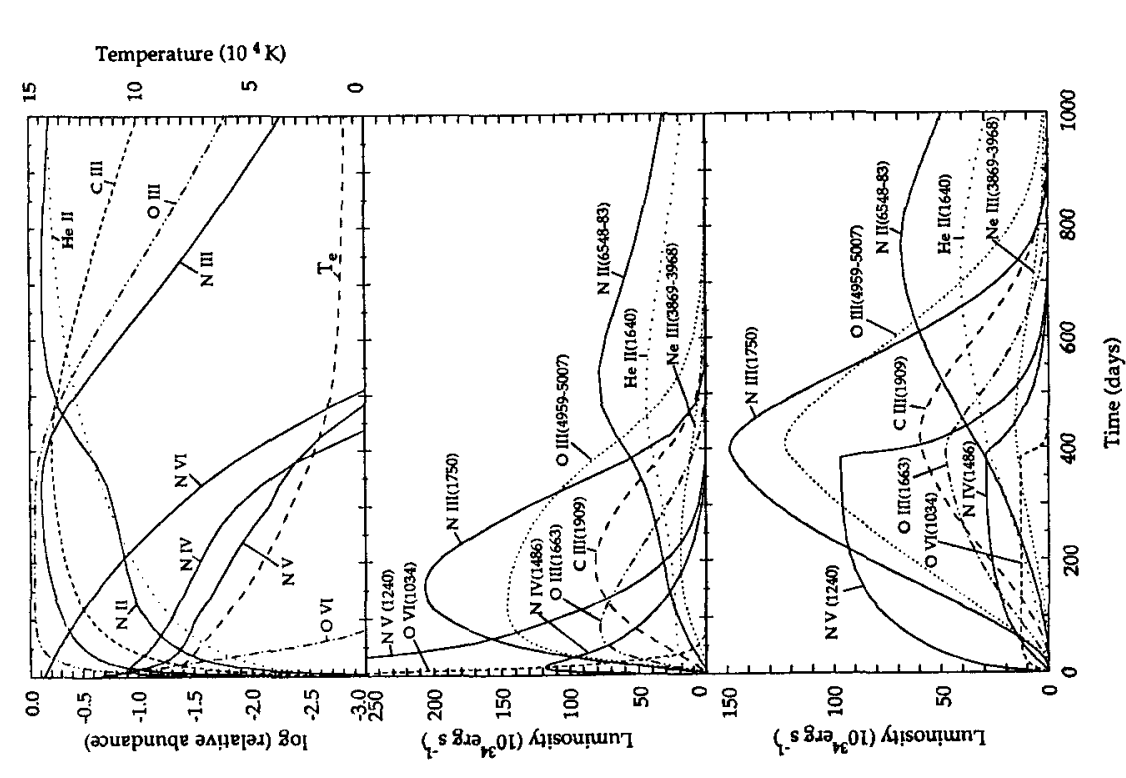

롫

施

总 莺

焉

i $\frac{5}{\infty}=$

称产

要宦

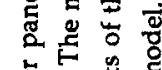

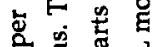
윽 융 잉

正要

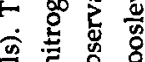

蛋

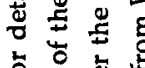

s

区ั

造

$<$ 원

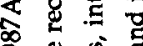

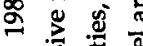

Z

Temperature $\left(10^{4} \mathrm{~K}\right)$

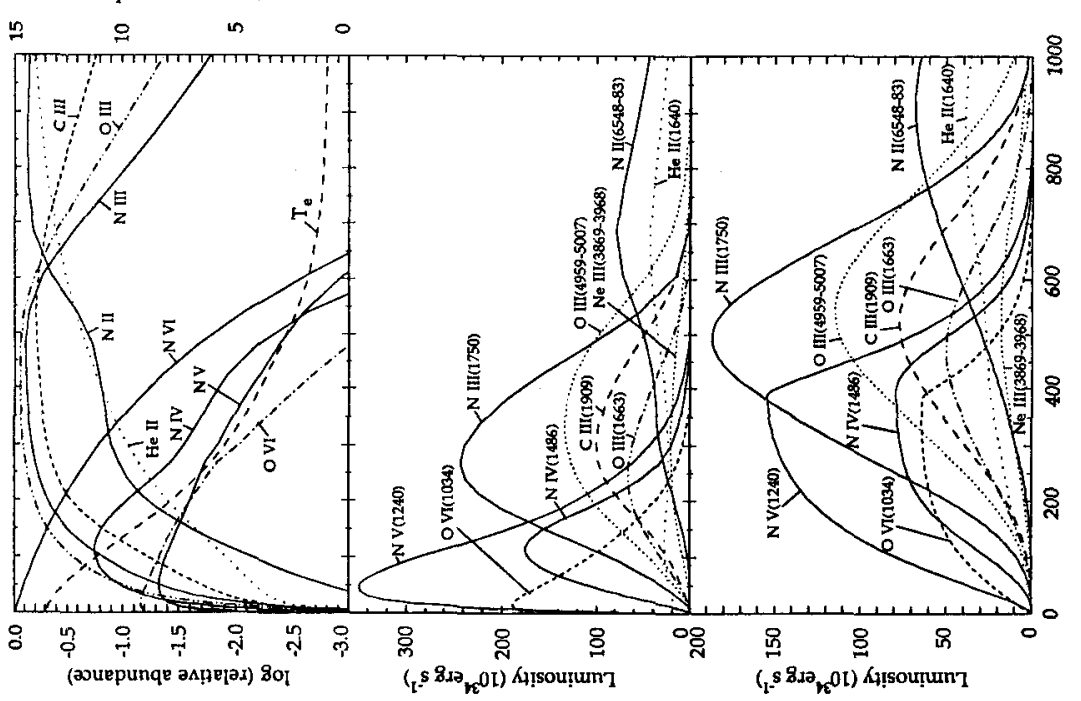

ह

$+\infty \Xi$

范至鸹

之

3 王

四 吾

형

조영

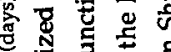

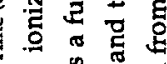

焉总起

䓃究苞

..

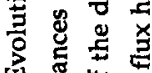

宊

ก

\%

事要焉 
VI. Recombination, however, sets in fairly rapidly and a population of $\mathrm{N} V$ builds up, which then recombines to N IV and N III. The rate at which recombination proceeds is directly determined by the density of the gas, since the recombination time $t_{r e c}=\left(\alpha_{r} n_{e}\right)^{-}$ 1 , where $\alpha_{\mathrm{r}}$ is the recombination coefficient. At a temperature of $10^{5} \mathrm{~K}$ and a density of

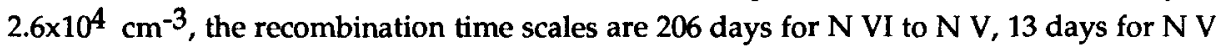
to $\mathrm{N}$ IV, 7 days for N IV to N III, and 14 days for N III to N II. These are thus short, or comparable, to the evolutionary time scale, and recombination is indeed important for the evolution. These time scales show that the gas would quickly become neutral if only recombination was involved. The gas temperature is, however, high enough for collisional ionization to prevent the gas from recombining more than to C III, N III etc., explaining why lines from these ions display the linear increase typical for a non-recombining ion. $\mathrm{N}$ IV, on the other hand, is less affected by collisional ionization and recombines, giving a roughly constant flux. This explains the qualitative behaviour of the observed fluxes in F89. The time scale determining the evolution is the longest of these time scales, i.e. the $\mathrm{N}$ $\mathrm{VI}$ to $\mathrm{N} \mathrm{V}$ recombination time scale. The cooling also occurs on this time scale, and proceeds rather smoothly. At $\sim 300$ days the temperature is $\sim 5.5 \times 10^{4} \mathrm{~K}$, close to that determined from the [O III] lines. The ionic abundances of C III, N III and O III are all similar, until $\sim 600$ days. This is due to the importance of collisional ionization, which for these ions give a situation close to the coronal case. When the temperature decreases below $\sim 3 \times 10^{4} \mathrm{~K}$ recombination of $\mathrm{N}$ III etc. sets in, and the different ions evolve differently on their respective recombination time scale. This calculation thus justifies the relations between the ionic and total abundances in F89. The middle panel of Fig. 1 shows the instantaneous values of the emission in the different lines. The $N$ III] line e.g. has a very broad emissivity peak, due to the slow recombination, giving a nearly linear increase in the observed flux (lower panel). The emissivity of N IV] $\lambda 1486$ is more concentrated to the early stages, and thus has a more constant observed flux.

Since the initial state of the gas is determined by the ionizing burst, the lines give constraints on this. In Fig. 2 we show an analogous calculation for the Woosley 10L model. Because of the lower radiation temperature $\left(2 \times 10^{5} \mathrm{~K}\right)$, the initial ionization and temperature is lower, giving a more rapid recombination and thus a "flatter" light curve for $\mathrm{N} \mathrm{V}$. Also the final decay is faster. In order to reproduce the observed state of ionization, the spectrum must contain a sufficient number of photons above $\sim 100 \mathrm{eV}$. This implies that the peak temperature must be more than $\sim(3-6) \times 10^{5} \mathrm{~K}$. The Shigeyama et al. 11E1Y6 model reproduces the increasing part well, while Woosley's models appear to have too low a peak temperature to reproduce the initial rise. This illustrates how these models can give unique information on the unobserved, but important early stages of the explosion.

We have also calculated models with different densities. Since both the cooling and recombination are governed by this, the time evolution and line ratios are sensitive to the density. It is encouraging that a density of $(2-3) \times 10^{4} \mathrm{~cm}^{-3}$, very close to that derived from 
the C III] diagnosis, gives the best agreement with the observations. The total mass in the shell is somewhat sensitive to the ionizing spectrum, with a typical range of $0.02-0.05$ $\mathrm{M}_{\mathrm{o}}$.

It is of great interest to determine the absolute metallicity, as well as the $\mathrm{He} / \mathrm{H}$ ratio. Unfortunately, the errors in the observed $\mathrm{H} \beta$ and He II $\lambda 4686$ fluxes are quite large, so the results should be taken with some caution. Preliminary results, however, suggest that the total $\mathrm{CNO}$ abundance is $0.2-0.3$ times solar, while the $\mathrm{He} / \mathrm{H}$ ratio is uncertain, but in the range $0.1-0.2$, by number. The $N / C$ and $N / O$ ratios are for these models $\sim 5$ and $\sim 2$, respectively, confirming that the assumptions in $\mathrm{F} 89$ are reasonable approximations. These are within the errors given in F89.

Except for the observed UV, and optical [O III], He II and Balmer lines, there are also other lines that may be observable. In the far UV the C III $\lambda 990$ and N III $\lambda 970$ resonance lines are both strong. In the same way as the C IV $\lambda 1550$ line they may, however, be unobservable due to interstellar absorption. The $O$ VI $\lambda 1034$ line is especially sensitive to the peak temperature of the radiation (Figs. 1 and 2). The possibility of actually observing this (Shull, priv. comm.) is thus extremely interesting. In the optical the [N II] $\lambda \lambda 6548-83,5755$ lines are expected to become strong after $\sim 300$ days. This coincides with the recombination from $\mathrm{N}$ III to $\mathrm{N}$ II. Also the [Ne III] $\lambda \lambda 3869-3968$ lines can be fairly strong, as well as at late times [O II] $\lambda \lambda 3726-29$.

\section{Discussion}

The observations of the narrow emission lines of SN 1987A have given us direct information about the structure of the circumstellar medium, which is of great importance for understanding the history of the progenitor. The radio observations by Turtle et al. (1987) were interpreted by Chevalier and Fransson (1987) as evidence for a wind with mass loss of $6 \times 10^{-6} \mathrm{M}_{\mathrm{o}} \mathrm{yr}^{-1}$ for a wind velocity of $550 \mathrm{~km} \mathrm{~s}^{-1}$. The density of this gas at $\sim 5 \times 10^{17} \mathrm{~cm}$ is, however, far too small to explain these observations. The dense gas we observe in these emission lines probably only constitute a minor fraction of all the gas around the supernova. The total number of ionizing photons is $\sim 10^{57}$ from the supernova, enough to ionize $\sim 0.8 \mathrm{M}_{0}$, i.e. roughly 15-40 times that of the shell. The gas inside the shell is mainly shocked gas from the blue stellar wind and does not absorb many of the photons from the supernova. The gas outside the shell is due to the red supergiant (RSG) wind, with a density of $\sim 20\left(\mathrm{r} / 10^{18} \mathrm{~cm}\right)^{-2}\left(\ddot{M} / 10^{-5} \mathrm{M}_{\mathrm{o}} \mathrm{yr}^{-1}\right) \mathrm{cm}^{-3}$. Here $\dot{\mathrm{M}}$ is the mass loss rate of the RSG. Of this gas, $\sim 0.8 \mathrm{M}_{\mathrm{o}}$ will be ionized by the burst in a way similar to the shell, but then recombine on a much longer time scale $\left(\sim 10^{3}\right.$ years for $\mathrm{N} V I$ to $\left.\mathrm{N} V\right)$. In addition, the radiation from the blue progenitor will ionize the hydrogen in the RSG wind, as long as $\dot{M}$ is less than $\sim 2 \times 10^{-5}\left(R_{S} / 10^{18} \mathrm{~cm}\right)^{1 / 2} \mathrm{M}_{\mathrm{o}} \mathrm{yr}^{-1}$. Since the emission measure of this gas is a factor of $\sim 25\left(\dot{\mathrm{M}} / 10^{-5} \mathrm{M}_{\circ} \mathrm{yr}^{-1}\right)^{-2}$ smaller, emission from this gas 
will be difficult to observe. The column density in ions like C IV, N V and Si IV etc. may, however, be large enough $\left(10^{14}-10^{15} \mathrm{~cm}^{-2}\right)$ to contribute to the absorptions observed in the early spectra (de Boer et al. 1987; Dupree et al. 1987). Also from radio observations it is difficult to obtain any constraints on the total amount of gas lost by the progenitor. The free-free optical depth at a wavelength $\lambda$ is given by $\tau_{\mathrm{ff}}=6.1 \times 10^{-30} \lambda^{2} \mathrm{~T}_{5}^{-1.5} \mathrm{EM}$, where $\mathrm{EM}$ is the emission measure and $\mathrm{T}_{5}$ the gas temperature in $10^{5} \mathrm{~K}$. For a RSG wind with inner boundary $R_{i n}$ and a velocity of $10 \mathrm{~km} \mathrm{~s}^{-1}$, the emission measure of the gas is only $\sim 1.8 \times 10^{20}\left(\dot{\mathrm{M}} / 10^{-5} \mathrm{M}_{\mathrm{o}} \mathrm{yr}^{-1}\right)^{2}\left(\mathrm{R}_{\mathrm{in}} / 10^{18} \mathrm{~cm}\right)^{-3} \mathrm{~cm}^{-5}$. It is thus easy to "hide" several solar masses of circumstellar gas, lost in the main sequence and red supergiant phases.

Acknowledgements: We have benefitted from discussions with Roger Chevalier, Ken Nomoto, Mike Shull, and Joe Wampler. This research has been supported by grants from the Swedish Natural Science Research Council and the Royal Swedish Academy of Sciences.

\section{REFERENCES}

Arnett, W.D. 1988, in Proc. of the George Mason Conference on SN 1987A, in press. Chevalier, R.A., and Fransson, C. 1987, Nature, $\underline{328}, 44$.

de Boer, K.S., Grewing, M., Richtler, T., Wamsteker, W., Gry, C., and Panagia, N. 1987, Astron. Astrophys. 177, L37.

Dupree, A.K., Kirshner, R.P., Nassiopoulos, G.E., Raymond, J.C., and Sonneborn, G. 1987, Ap. J. $\underline{320}, 597$.

Fransson, C., and Lundqvist, P. 1988, in preparation.

Fransson, C., Cassatella, A., Gilmozzi, R., Kirshner, R.P., Panagia, N., Sonneborn, G., and Wamsteker, W. 1989, Ap. J., Jan. 1 (F89).

Lundqvist, P., and Fransson, C. 1987, in Proc. of the ESO Workshop on SN 1987A, ed. I.J. Danziger, p. 495.

Lundqvist, P., and Fransson, C. 1989, in preparation.

Sonneborn, G., et al., IAU Circ.

Shigeyama, T., Nomoto, K., and Hashimoto, M. 1988, Astron. Astrophys. 196, 141.

Turtle, A.J., Campbell-Wilson, D., Bunton, J.D., Jauncey, D.L., Kesteven, M.J., Manchester, R.N., Norris, R.P., Storey, M.C., and Reynolds, J.E. 1987, Nature , 327, 38.

Wampler, E.J., and Richichi, A. 1988, submitted to Astron. Astrophys.

Woosley, S.E. 1988, Ap. J. 330, 218. 